\title{
Global convergence of a modified conjugate gradient method
}

Xuesha Wu*

"Correspondence: wuxuesha2013@126.com

College of General Education, Chongqing College of Electronic Engineering, Chongqing, 401331 P.R. China
(C2014 Wu; licensee Springer. This is an Open Access article distributed under the terms of the Creative Commons Attribution License (http://creativecommons.org/licenses/by/2.0), which permits unrestricted use, distribution, and reproduction in any medium, provided the original work is properly cited.

\section{Introduction}

The nonlinear conjugate gradient method is one of the best methods to solve unconstrained optimization problems. It comprises a class of unconstrained optimization algorithms which is characterized by low memory requirements and strong local or global convergence properties. Therefore, a modified nonlinear conjugate gradient method is proposed and analyzed in this paper.

Consider the following unconstrained optimization problem:

$$
\min _{x \in R^{n}} f(x)
$$

where $f: R^{n} \rightarrow R$ is a smooth function and its gradient is denoted by $g$.

The conjugate gradient methods for solving the above problem often use the following iterative rules:

$$
x_{k+1}=x_{k}+\alpha_{k} d_{k}
$$

where $x_{k}$ is the current iterate, the stepsize $\alpha_{k}$ is a positive scalar which is generated by some line search, and the search direction $d_{k}$ is defined by

$$
d_{k}= \begin{cases}-g_{k}, & \text { for } k=1 ; \\ -g_{k}+\beta_{k} d_{k-1}, & \text { for } k \geq 2,\end{cases}
$$

where $g_{k}=\nabla f\left(x_{k}\right), \beta_{k}$ is the conjugate parameter which determines the performances of the corresponding methods. There are many well-known parameters $\beta_{k}$, such as

$$
\beta_{k}^{\mathrm{PRP}}=\frac{g_{k}^{T}\left(g_{k}-g_{k-1}\right)}{\left\|g_{k-1}\right\|^{2}} \quad(\text { Polak-Ribière-Polyak (PRP) }[1,2]),
$$




$$
\begin{aligned}
& \beta_{k}^{\mathrm{LS}}=-\frac{g_{k}^{T}\left(g_{k}-g_{k-1}\right)}{d_{k-1}^{T} g_{k-1}} \quad \text { (Liu-Storey (LS) [3]), } \\
& \beta_{k}^{\mathrm{HZ}}=\left(y_{k-1}-2 d_{k-1} \frac{\left\|y_{k-1}\right\|^{2}}{d_{k-1}^{T} y_{k-1}}\right)^{T} \frac{g_{k}}{d_{k-1}^{T} y_{k-1}} \quad \text { (Hager-Zhang [4]), }
\end{aligned}
$$

where $\|\cdot\|$ is the Euclidean norm. Their corresponding methods are generally called PRP, LS, and HZ conjugate gradient methods. If $f$ is a strictly convex quadratic function, these methods are equivalent in the case that an exact line search is used. If $f$ is non-convex, their behaviors may show some differences.

When the objective function is convex, Polak and Ribière [1] proved that the PRP method is globally convergent under the exact line search. But Powell [5] showed that the PRP method does not converge globally for some non-convex functions. However, in the past few years, the PRP method is generally believed to be the most efficient conjugate gradient method in practical computation. One remarkable property of the PRP method is that it essentially performs a restart if a bad direction occurs (see [6]). But Powell [5] constructed an example showing that the PRP method can cycle infinitely without approaching any stationary point even if an exact line search is used. This counter-example also indicates that the PRP method has a drawback in that it may not globally be convergent when the objective function is non-convex. Recently, Zhang et al. [7] proposed a descent modified PRP conjugate gradient method and proved its global convergence. The LS method has a similar property as the PRP method. The global convergence of the LS method with the Grippo-Lucidi line search has also been proved in [8]. Some researchers have further studied the LS method (see Liu [9], Liu and Du [10]). In addition, Hager and Zhang [4] gave another effective method, namely the CG-DESCENT method. It not only has stable convergence, but it also shows an effective numerical experiment result. In this method, the parameter $\beta_{k}$ is computed by $\beta_{k}=\max \left\{\beta_{k}^{\mathrm{HZ}}, \eta_{k}\right\}$, where $\eta_{k}=\frac{-1}{\left\|d_{k-1}\right\| \min \left\{\eta,\left\|g_{k-1}\right\|\right\}}$, $\eta>0$.

In the next section, a modified conjugate gradient method is proposed. In Section 3, we prove the global convergence of the proposed method for non-convex functions in the case of the strong Wolfe line search. In Section 4, we report some numerical results.

\section{The new algorithm}

Recently, some people have studied some variants of the LS method. For example, Li et al. [11] proposed a modified LS method where the parameter $\beta_{k}$ is computed by

$$
\beta_{k}=-\frac{g_{k}^{T}\left(g_{k}-g_{k-1}\right)}{d_{k-1}^{T} g_{k-1}}-t \frac{\left\|g_{k}-g_{k-1}\right\|^{2} d_{k-1}^{T} g_{k}}{\left(d_{k-1}^{T} g_{k-1}\right)^{2}}
$$

where $t>\frac{1}{4}$ is a constant. They proved the global convergence of the modified method with the Armijo line search and Wolfe line search. Tang et al. [12] proved the LS method with the new line search. Liu et al. [13] studied a modified LS method where the parameter $\beta_{k}$ is computed by

$$
\beta_{k}^{\mathrm{LS} 2}= \begin{cases}\frac{g_{k}^{T}\left(g_{k}-g_{k-1}\right)}{\rho\left|g_{k}^{T} d_{k-1}\right|-g_{k-1}^{T} d_{k-1}} & \text { if } \min \{1, \rho-1-\xi\} \cdot\left\|g_{k}\right\|^{2}>\left|g_{k}^{T} g_{k-1}\right|, \\ 0 & \text { else, }\end{cases}
$$


where $\rho>1+\xi, \xi>0$. They proved the global convergence of the corresponding method with the Wolfe line search. In 2006, Wei et al. [14] proposed a modified PRP method where the parameter $\beta_{k}$ is obtained by

$$
\beta_{k}=\frac{g_{k}^{T}\left(g_{k}-\frac{\left\|g_{k}\right\|}{\left\|g_{k-1}\right\|} g_{k-1}\right)}{\left\|g_{k-1}\right\|^{2}} .
$$

They proved its global convergence with the exact line search, the strong Wolfe line search, and the Grippo-Lucidi line search, respectively. Their work overcomes the weak convergence of the PRP method. Inspired by their work, we consider a variant of LS method, i.e.

$$
\beta_{k}^{\mathrm{VLS}}=\frac{g_{k}^{T}\left(g_{k}-t_{k} g_{k-1}\right)}{-\lambda d_{k-1}^{T} g_{k-1}+(1-\lambda) \max \left\{0, g_{k}^{T} d_{k-1}\right\}},
$$

where $t_{k}=\frac{\left\|g_{k}\right\|}{\left\|g_{k-1}\right\|}, \lambda \in(0,1)$ and $\lambda>2 \sigma$. Obviously, the denominator of (2.1) is a convex combination of $-d_{k-1}^{T} g_{k-1}$ and $\max \left\{0, g_{k}^{T} d_{k-1}\right\}$ which may avoid the denominator of $\beta_{k}^{\mathrm{LS}}$ tending to zero. Now, we state formally the corresponding algorithm scheme for unconstrained optimization problems.

\section{Algorithm 2.1}

Step 0: Given an initial $x_{1} \in R^{n}, \varepsilon \geq 0, \lambda=0.8$. Set $k=1$.

Step 1: If $\left\|g_{1}\right\| \leq \varepsilon$, then stop.

Step 2: Compute $\alpha_{k}$ by the strong Wolfe line search $\left(0<\delta<\sigma<\frac{1}{2}\right)$ :

$$
\begin{gathered}
f\left(x_{k}+\alpha_{k} d_{k}\right) \leq f\left(x_{k}\right)+\delta \alpha_{k} g_{k}^{T} d_{k}, \\
\left|g\left(x_{k}+\alpha_{k} d_{k}\right)^{T} d_{k}\right| \leq-\sigma g_{k}^{T} d_{k} .
\end{gathered}
$$

Step 3: Let $x_{k+1}=x_{k}+\alpha_{k} d_{k}, g_{k+1}=g\left(x_{k+1}\right)$, if $\left\|g_{k+1}\right\| \leq \varepsilon$, then stop.

Step 4: Compute $\beta_{k+1}$ by (2.1), and generate $d_{k+1}$ by (1.3).

Step 5: Set $k=k+1$, go to step 2 .

In some references, the sufficient descent condition

$$
g_{k}^{T} d_{k} \leq-c\left\|g_{k}\right\|^{2}, \quad c>0,
$$

is always assumed to hold. Because it plays an important role in proving the global convergence of conjugate gradient methods. Fortunately, in this paper, the search direction $d_{k}$ satisfies the sufficient descent condition in the case of the strong Wolfe line search without any assumption.

Lemma 2.1 Let the sequences $\left\{g_{k}\right\}$ and $\left\{d_{k}\right\}$ be generated by Algorithm 2.1, then we obtain

$$
g_{k}^{T} d_{k} \leq-\left(1-\frac{2 \sigma}{\lambda}\right) \cdot\left\|g_{k}\right\|^{2}
$$

Proof The conclusion can be proved by induction. Since $g_{1}^{T} d_{1}=-\left\|g_{1}\right\|^{2}$, the conclusion (2.5) holds for $k=1$. Now we assume that the conclusion (2.5) holds for $k \geq 1$ and $g_{k+1} \neq 0$. 
One gets from (1.3) that

$$
\begin{aligned}
-\frac{g_{k+1}^{T} d_{k+1}}{\left\|g_{k+1}\right\|^{2}} & =1-\beta_{k+1}^{\mathrm{VLS}} \frac{g_{k+1}^{T} d_{k}}{\left\|g_{k+1}\right\|^{2}} \geq 1-\left|\beta_{k+1}^{\mathrm{VLS}}\right| \cdot \frac{\left|g_{k+1}^{T} d_{k}\right|}{\left\|g_{k+1}\right\|^{2}} \\
& \geq 1-\frac{\left\|g_{k+1}\right\|^{2}+\frac{\left\|g_{k+1}\right\|}{\left\|g_{k}\right\|} \cdot\left|g_{k+1}^{T} g_{k}\right|}{\lambda\left|g_{k}^{T} d_{k}\right|} \cdot \frac{\left|g_{k+1}^{T} d_{k}\right|}{\left\|g_{k+1}\right\|^{2}} \\
& \geq 1-\frac{2\left\|g_{k+1}\right\|^{2}}{\lambda\left|g_{k}^{T} d_{k}\right|} \cdot \frac{\sigma\left|g_{k}^{T} d_{k}\right|}{\left\|g_{k+1}\right\|^{2}}=1-\frac{2 \sigma}{\lambda} .
\end{aligned}
$$

From the above inequality, the conclusion (2.5) holds for $k+1$. Thus, the conclusion (2.5) holds for $k \in N^{+}$.

Remark 2.1 From (2.5) and the definition of $\beta_{k}^{\mathrm{VLS}}$, it is not difficult to find that

$$
\begin{aligned}
\beta_{k}^{\mathrm{VLS}} & =\frac{g_{k}^{T}\left(g_{k}-t_{k} g_{k-1}\right)}{-\lambda d_{k-1}^{T} g_{k-1}+(1-\lambda) \max \left\{0, g_{k}^{T} d_{k-1}\right\}} \\
& \geq \frac{\left\|g_{k}\right\|^{2}-\frac{\left\|g_{k}\right\|}{\left\|g_{k-1}\right\|} \cdot\left|g_{k}^{T} g_{k-1}\right|}{-\lambda d_{k-1}^{T} g_{k-1}+(1-\lambda) \max \left\{0, g_{k}^{T} d_{k-1}\right\}} \\
& \geq \frac{\left\|g_{k}\right\|^{2}-\frac{\left\|g_{k}\right\|}{\left\|g_{k-1}\right\|} \cdot\left\|g_{k}^{T}\right\| \cdot\left\|g_{k-1}\right\|}{-\lambda d_{k-1}^{T} g_{k-1}+(1-\lambda) \max \left\{0, g_{k}^{T} d_{k-1}\right\}}=0 .
\end{aligned}
$$

\section{Global convergence of Algorithm 2.1}

In order to prove the global convergence of Algorithm 2.1, the following assumptions for the objective function are often used.

\section{Assumption (H)}

(i) The level set $\Omega=\left\{x \mid f(x) \leq f\left(x_{1}\right)\right\}$ is bounded, where $x_{1}$ is the starting point.

(ii) In some neighborhood $V$ of $\Omega$, the objective function $f$ is continuously differentiable, and its gradient is Lipschitz continuous, i.e., there exists a constant $L>0$ such that

$$
\|g(x)-g(y)\| \leq L\|x-y\|, \quad \text { for all } x, y \in V .
$$

From Assumption $(\mathrm{H})$, there exists a constant $\tilde{r}>0$ such that

$\left\|g_{k}\right\| \leq \tilde{r}, \quad$ for all $k$.

The conclusion of the following lemma, often called the Zoutendijk condition, is usually used to prove the global convergence properties of conjugate gradient methods. It was originally established by Zoutendijk [15].

Lemma 3.1 Suppose Assumption (H) holds. Let the sequences $\left\{g_{k}\right\}$ and $\left\{d_{k}\right\}$ be generated by Algorithm 2.1, then we have

$$
\sum_{k \geq 1} \frac{\left(g_{k}^{T} d_{k}\right)^{2}}{\left\|d_{k}\right\|^{2}}<+\infty
$$


Lemma 3.2 Suppose Assumption (H) holds. Let the sequences $\left\{g_{k}\right\}$ and $\left\{d_{k}\right\}$ be generated by Algorithm 2.1, and let there exist a constant $r>0$ such that

$\left\|g_{k}\right\| \geq r, \quad$ for all $k \geq 1$.

Then we have

$$
\sum_{k \geq 2}\left\|u_{k}-u_{k-1}\right\|^{2}<+\infty, \quad u_{k}=\frac{d_{k}}{\left\|d_{k}\right\|}
$$

Proof This lemma can be proved in a similar way as in [16], so we omit it.

Lemma 3.3 Suppose Assumption $(\mathrm{H})$ holds. Let the sequences $\left\{g_{k}\right\}$ and $\left\{d_{k}\right\}$ be generated by Algorithm 2.1, and let the sequence $\left\{g_{k}\right\}$ satisfy

$$
0<r \leq\left\|g_{k}\right\| \leq \tilde{r}, \quad \text { for all } k \geq 1 .
$$

Then the conjugate parameter $\beta_{k}^{\mathrm{VLS}}$ has property $(*)$, i.e.,

(1) there exists a constant $b>1$ such that $\left|\beta_{k}^{\mathrm{VLS}}\right| \leq b$;

(2) there exists a constant $\tau>0$, such that $\left\|x_{k}-x_{k-1}\right\| \leq \tau \Rightarrow\left|\beta_{k}^{\mathrm{VLS}}\right| \leq \frac{1}{2 b}$.

Proof It follows from (2.1), (3.4), and (2.5) that

$$
\begin{aligned}
\left|\beta_{k}^{\mathrm{VLS}}\right| & =\left|\frac{g_{k}^{T}\left(g_{k}-t_{k} g_{k-1}\right)}{-\lambda d_{k-1}^{T} g_{k-1}+(1-\lambda) \max \left\{0, g_{k}^{T} d_{k-1}\right\}}\right| \\
& \leq \frac{\left\|g_{k}\right\|\left(\left\|g_{k}\right\|+\frac{\tilde{r}}{r} \cdot\left\|g_{k-1}\right\|\right)}{\lambda\left|d_{k-1}^{T} g_{k-1}\right|} \\
& \leq \frac{\left\|g_{k}\right\|\left(\left\|g_{k}\right\|+\frac{\tilde{r}}{r} \cdot\left\|g_{k-1}\right\|\right)}{(\lambda-2 \sigma)\left\|g_{k-1}\right\|^{2}} \leq \frac{\tilde{r}\left(\tilde{r}+\frac{\tilde{r}^{2}}{r}\right)}{(\lambda-2 \sigma) r^{2}} \\
& \leq \frac{\tilde{r}^{2}(r+\tilde{r})}{(\lambda-2 \sigma) r^{3}}=b .
\end{aligned}
$$

Define $\tau=\frac{(\lambda-2 \sigma) r^{2}}{4 L \tilde{r} b}$. Let $\left\|x_{k}-x_{k-1}\right\| \leq \tau$, it then follows from Assumption (H)(ii) that

$$
\begin{aligned}
\left|\beta_{k}^{\mathrm{VLS}}\right| & =\left|\frac{g_{k}^{T}\left(g_{k}-t_{k} g_{k-1}\right)}{-\lambda d_{k-1}^{T} g_{k-1}+(1-\lambda) \max \left\{0, g_{k}^{T} d_{k-1}\right\}}\right| \\
& \leq \frac{\left\|g_{k}\right\|\left(\left\|g_{k}-g_{k-1}\right\|+\left\|g_{k-1}-t_{k} g_{k-1}\right\|\right)}{\lambda\left|d_{k-1}^{T} g_{k-1}\right|} \\
& \leq \frac{\tilde{r}\left(L \tau+\left\|g_{k-1}-t_{k} g_{k-1}\right\|\right)}{\lambda\left|d_{k-1}^{T} g_{k-1}\right|} \leq \frac{\tilde{r}\left(L \tau+\mid\left\|g_{k}\right\|-\left\|g_{k-1}\right\|\right)}{(\lambda-2 \sigma)\left\|g_{k-1}\right\|^{2}} \\
& \leq \frac{\tilde{r}\left(L \tau+\left\|g_{k}-g_{k-1}\right\|\right)}{(\lambda-2 \sigma)\left\|g_{k-1}\right\|^{2}} \leq \frac{2 L \tau \tilde{r}}{(\lambda-2 \sigma) r^{2}}=\frac{1}{2 b} .
\end{aligned}
$$

Lemma 3.4 Suppose Assumption (H) holds. Consider any method of (1.2)-(1.3), where $\beta_{k} \geq$ 0 , and where $\alpha_{k}$ satisfies the strong Wolfe line search. If $\beta_{k}$ has the property (*), and (2.5) 
and (3.4) hold, then there exists a constant $\tau>0$, for any $\Delta \in Z^{+}$and $k_{0} \in Z^{+}$, and for any $k \geq k_{0}$ such that

$$
\left|\Re_{k, \Delta}^{\tau}\right|>\frac{\Delta}{2},
$$

where $\mathfrak{R}_{k, \Delta}^{\tau} \triangleq\left\{i \in Z^{+}: k \leq i \leq k+\Delta-1,\left\|x_{i}-x_{i-1}\right\| \geq \tau\right\},\left|\Re_{k, \Delta}^{\tau}\right|$ denotes the number of $\Re_{k, \Delta}^{\tau}$.

Proof This lemma plays an important role in proving the global convergences of PRP, HS, and LS conjugate gradient methods, and so on. It was originally proved in [17]. From Remark 2.1 and Lemma 3.3, it is easy to find that Algorithm 2.1 leads to the conclusion of Lemma 3.4.

Theorem 3.1 Suppose Assumption $(\mathrm{H})$ holds. Let the sequences $\left\{g_{k}\right\}$ and $\left\{d_{k}\right\}$ be generated by Algorithm 2.1. If $\beta_{k}^{\mathrm{VLS}}$ has the property $(*)$, and (2.5) holds, then we obtain

$$
\liminf _{k \rightarrow+\infty}\left\|g_{k}\right\|=0
$$

Proof Using mathematical induction. Suppose that (3.5) does not hold, which means that there exists $r>0$ such that

$$
\left\|g_{k}\right\| \geq r, \quad \text { for all } k \geq 1 \text {. }
$$

We also define $u_{k}=\frac{d_{k}}{\left\|d_{k}\right\|}$, then for all $l, k \in Z^{+}(l \geq k)$, we have

$$
\begin{aligned}
x_{l}-x_{k-1} & =\sum_{i=k}^{l}\left\|x_{i}-x_{i-1}\right\| \cdot u_{i-1} \\
& =\sum_{i=k}^{l}\left\|s_{i-1}\right\| \cdot u_{k-1}+\sum_{i=k}^{l}\left\|s_{i-1}\right\|\left(u_{i-1}-u_{k-1}\right),
\end{aligned}
$$

where $s_{i-1}=x_{i}-x_{i-1}$.

From Assumption (H), we know that there exists a constant $\xi>0$ such that

$$
\|x\| \leq \xi, \quad \text { for } x \in V
$$

By (3.7), we have

$$
\sum_{i=k}^{l}\left\|s_{i-1}\right\| \cdot u_{k-1}=\left(x_{l}-x_{k-1}\right)-\sum_{i=k}^{l}\left\|s_{i-1}\right\|\left(u_{i-1}-u_{k-1}\right) .
$$

Since (3.8) and (3.9) hold, we have

$$
\sum_{i=k}^{l}\left\|s_{i-1}\right\| \leq 2 \xi+\sum_{i=k}^{l}\left\|s_{i-1}\right\| \cdot\left\|u_{i-1}-u_{k-1}\right\| .
$$

Let $\tau$ come from Lemma 3.4, and we define $\Delta=[8 \xi / \tau]$, where $8 \xi / \tau \leq \Delta<(8 \xi / \tau)+1$, and $\Delta \in Z^{+}$. 
From Lemma 3.2, we know that there exists $k_{0}$ such that

$$
\sum_{i \geq k_{0}}\left\|u_{i+1}-u_{i}\right\|^{2} \leq \frac{1}{4 \Delta}
$$

From the Cauchy-Schwarz inequality and (3.11), and letting $\forall i \in[k, k+\Delta-1]$, we have

$$
\begin{aligned}
\left\|u_{i-1}-u_{k-1}\right\| & \leq \sum_{j=k}^{i-1}\left\|u_{j}-u_{j-1}\right\| \\
& \leq(i-k)^{\frac{1}{2}}\left(\sum_{j=k}^{i-1}\left\|u_{j}-u_{j-1}\right\|^{2}\right)^{\frac{1}{2}} \\
& \leq \Delta^{\frac{1}{2}} \cdot\left(\frac{1}{4 \Delta}\right)^{\frac{1}{2}}=\frac{1}{2} .
\end{aligned}
$$

From Lemma 3.4, we know that there exists $k \geq k_{0}$ such that

$$
\left|\Re_{k, \Delta}^{\tau}\right|>\frac{\Delta}{2}
$$

By (3.10), (3.12), and (3.13), we have

$$
2 \xi \geq \frac{1}{2} \sum_{i=k}^{k+\Delta-1}\left\|s_{i-1}\right\|>\frac{\tau}{2}\left|\Re_{k, \Delta}^{\tau}\right|>\frac{\tau \Delta}{4} .
$$

From (3.14), we have $\Delta<8 \xi / \tau$, which is a contradiction with the definition of $\Delta$. Therefore,

$$
\liminf _{k \rightarrow+\infty}\left\|g_{k}\right\|=0
$$

Thus we complete the proof of Theorem 3.1.

\section{Numerical results}

In this section, we compare the performance of Algorithm 2.1 with those of the PRP+ method [18] and the CG-DESCENT method [4] in the number of function evaluations and CPU time in seconds with the strong Wolfe line search. The test problems are some large-scaled unconstrained optimization problems in $[19,20]$. The parameters in the line search are chosen as follows: $\delta=0.01, \sigma=0.1$. If $\left\|g_{k}\right\|_{\infty} \leq 10^{-6}$ is satisfied, we will terminate the program. All codes were written in Fortran 6.0 and run on a PC with $2.0 \mathrm{GHz} \mathrm{CPU}$ processor and $512 \mathrm{MB}$ memory and Windows XP operation system.

The numerical results are reported in Table 1. The first column 'Problems' represents the problem's name in $[19,20]$. 'Dim' denotes the dimension of the test problems. The detailed numerical results are listed in the form NF $\backslash$ CPU, where NF and CPU denote the number of function evaluations and CPU time in seconds, respectively.

We say that, in particular for the $i$ th problem, the performance of the M1 method was better than the performance of M2 method, if the CPU time, or the number of function 
Table 1 The numerical results of Algorithm 2.1, PRP+ method and CG-DESCENT method

\begin{tabular}{|c|c|c|c|c|}
\hline Problems & $\operatorname{Dim}$ & Algorithm 2.1 & PRP+ method & CG-DESCENT method \\
\hline \multirow[t]{2}{*}{ Extended Freudenstein \& Roth } & 5,000 & $58 \backslash 0.07$ & $1,144 \backslash 0.09$ & $13,235 \backslash 1.24$ \\
\hline & 10,000 & $12 \backslash 0.01$ & $426 \backslash 0.07$ & $23 \backslash 0.01$ \\
\hline \multirow[t]{2}{*}{ Extended Trigonometric } & 5,000 & $30 \backslash 0.07$ & $61 \backslash 0.08$ & $79 \backslash 0.10$ \\
\hline & 10,000 & $33 \backslash 0.19$ & $112 \backslash 0.28$ & $161 \backslash 0.40$ \\
\hline \multirow[t]{2}{*}{ Extended Rosenbrock } & 5,000 & $41 \backslash 0.02$ & $67 \backslash 0.02$ & $60 \backslash 0.02$ \\
\hline & 10,000 & $34 \backslash 0.03$ & $62 \backslash 0.03$ & $57 \backslash 0.01$ \\
\hline \multirow[t]{2}{*}{ Extended White \& Holst } & 5,000 & $40 \backslash 0.02$ & $53 \backslash 0.02$ & $45 \backslash 0.02$ \\
\hline & 10,000 & $38 \backslash 0.01$ & $43 \backslash 0.01$ & $52 \backslash 0.02$ \\
\hline \multirow[t]{2}{*}{ Extended Beale } & 5,000 & $15 \backslash 0.01$ & $26 \backslash 0.02$ & $24 \backslash 0.00$ \\
\hline & 10,000 & $15 \backslash 0.01$ & $26 \backslash 0.00$ & $24 \backslash 0.01$ \\
\hline \multirow[t]{2}{*}{ Extended Penalty } & 5,000 & $11 \backslash 0.01$ & $51 \backslash 0.00$ & $1,979 \backslash 0.17$ \\
\hline & 10,000 & $18 \backslash 0.02$ & $36 \backslash 0.02$ & $50 \backslash 0.02$ \\
\hline \multirow[t]{2}{*}{ Perturbed Quadratic } & 5,000 & $705 \backslash 0.35$ & $1,462 \backslash 0.42$ & $1,471 \backslash 0.36$ \\
\hline & 10,000 & $1,353 \backslash 0.88$ & $2,059 \backslash 1.19$ & $2,014 \backslash 0.95$ \\
\hline \multirow{2}{*}{ Raydan 2} & 5,000 & $9 \backslash 0.00$ & $9 \backslash 0.00$ & $9 \backslash 0.00$ \\
\hline & 10,000 & $9 \backslash 0.02$ & $9 \backslash 0.02$ & $9 \backslash 0.02$ \\
\hline \multirow[t]{2}{*}{ Diagonal 2} & 5,000 & $432 \backslash 0.44$ & $987 \backslash 0.67$ & $699 \backslash 0.45$ \\
\hline & 10,000 & $595 \backslash 1.28$ & $1,117 \backslash 1.52$ & $1,209 \backslash 1.60$ \\
\hline \multirow[t]{2}{*}{ Generalized Tridiagonal 1} & 5,000 & $42 \backslash 0.02$ & $2,013 \backslash 0.27$ & $53 \backslash 0.01$ \\
\hline & 10,000 & $71 \backslash 0.14$ & $578 \backslash 0.15$ & $707 \backslash 0.19$ \\
\hline \multirow[t]{2}{*}{ Extended Tridiagonal 1} & 5,000 & $12 \backslash 0.00$ & $23 \backslash 0.00$ & $28 \backslash 0.00$ \\
\hline & 10,000 & $12 \backslash 0.01$ & $28 \backslash 0.02$ & $29 \backslash 0.01$ \\
\hline \multirow[t]{2}{*}{ Extended Three Expo Terms } & 5,000 & $8 \backslash 0.01$ & $21 \backslash 0.01$ & $15 \backslash 0.02$ \\
\hline & 10,000 & $12 \backslash 0.04$ & $19 \backslash 0.05$ & $15 \backslash 0.03$ \\
\hline \multirow[t]{2}{*}{ Generalized Tridiagonal 2} & 5,000 & $50 \backslash 0.03$ & $94 \backslash 0.03$ & $95 \backslash 0.03$ \\
\hline & 10,000 & $62 \backslash 0.05$ & $97 \backslash 0.06$ & $77 \backslash 0.03$ \\
\hline \multirow[t]{2}{*}{ Diagonal 4} & 5,000 & $8 \backslash 0.00$ & $8 \backslash 0.00$ & $8 \backslash 0.00$ \\
\hline & 10,000 & $8 \backslash 0.00$ & $8 \backslash 0.00$ & $8 \backslash 0.00$ \\
\hline \multirow[t]{2}{*}{ Diagonal 5} & 5,000 & $9 \backslash 0.01$ & $9 \backslash 0.02$ & $9 \backslash 0.02$ \\
\hline & 10,000 & $9 \backslash 0.03$ & $9 \backslash 0.03$ & $9 \backslash 0.03$ \\
\hline Extended Himmelblau & 5,000 & $18 \backslash 0.00$ & $35 \backslash 0.00$ & $16 \backslash 0.00$ \\
\hline & 10,000 & $18 \backslash 0.02$ & $35 \backslash 0.03$ & $16 \backslash 0.00$ \\
\hline Generalized PSC1 & 5,000 & $1,886 \backslash 3.20$ & $633 \backslash 0.60$ & $17,679 \backslash 14.59$ \\
\hline & 10,000 & $729 \backslash 2.61$ & $1,271 \backslash 2.39$ & $8,364 \backslash 13.94$ \\
\hline Extended PSC1 & 5,000 & $17 \backslash 0.02$ & $13 \backslash 0.01$ & $16 \backslash 0.02$ \\
\hline & 10,000 & $17 \backslash 0.03$ & $15 \backslash 0.02$ & $16 \backslash 0.03$ \\
\hline Extended Powell & 5,000 & $46 \backslash 0.03$ & $138 \backslash 0.03$ & $250 \backslash 0.05$ \\
\hline & 10,000 & $74 \backslash 0.06$ & $88 \backslash 0.04$ & $311 \backslash 0.09$ \\
\hline Extended Block-Diagonal BD1 & 5,000 & $55 \backslash 0.02$ & $40 \backslash 0.01$ & $33 \backslash 0.01$ \\
\hline & 10,000 & $53 \backslash 0.06$ & $47 \backslash 0.05$ & $46 \backslash 0.05$ \\
\hline Extended Maratos & 5,000 & $71 \backslash 0.03$ & $132 \backslash 0.03$ & $103 \backslash 0.02$ \\
\hline & 10,000 & $69 \backslash 0.02$ & $96 \backslash 0.03$ & $99 \backslash 0.03$ \\
\hline Quadratic Diagonal Perturbed & 5,000 & $793 \backslash 0.22$ & $880 \backslash 0.22$ & $2,111 \backslash 0.39$ \\
\hline & 10,000 & $1,549 \backslash 0.62$ & $1,303 \backslash 0.66$ & $2,966 \backslash 1.16$ \\
\hline Extended Wood & 5,000 & $85 \backslash 0.02$ & $57 \backslash 0.01$ & $135 \backslash 0.01$ \\
\hline & 10,000 & $84 \backslash 0.04$ & $65 \backslash 0.03$ & $116 \backslash 0.05$ \\
\hline Extended Hiebert & 5,000 & $176 \backslash 0.04$ & $137 \backslash 0.03$ & $120 \backslash 0.03$ \\
\hline & 10,000 & $173 \backslash 0.05$ & $137 \backslash 0.05$ & $114 \backslash 0.03$ \\
\hline QuadraticQF1 & 5,000 & $1,222 \backslash 0.25$ & $1,854 \backslash 0.50$ & $1,397 \backslash 0.30$ \\
\hline & 10,000 & $1,396 \backslash 0.82$ & $1,864 \backslash 0.97$ & $2,545 \backslash 1.06$ \\
\hline Extended Quadratic Penalty QP2 & 5,000 & $45 \backslash 0.05$ & $80 \backslash 0.06$ & $76 \backslash 0.06$ \\
\hline & 10,000 & $43 \backslash 0.11$ & $71 \backslash 0.13$ & $84 \backslash 0.14$ \\
\hline QuadraticQF2 & 5,000 & $1,167 \backslash 0.40$ & $1,620 \backslash 0.44$ & $1,613 \backslash 0.36$ \\
\hline & 10,000 & $1,430 \backslash 1.14$ & $2,625 \backslash 1.45$ & $2,941 \backslash 1.31$ \\
\hline Extended EP1 & 5,000 & $6 \backslash 0.00$ & $6 \backslash 0.00$ & $6 \backslash 0.00$ \\
\hline & 10,000 & $413 \backslash 0.21$ & $513 \backslash 0.25$ & $439 \backslash 0.22$ \\
\hline Extended Tridiagonal 2 & 5,000 & $875 \backslash 0.23$ & $2,436 \backslash 0.27$ & $857 \backslash 0.11$ \\
\hline & 10,000 & $5,139 \backslash 1.17$ & $5,857 \backslash 1.29$ & $6,569 \backslash 1.47$ \\
\hline ARWHEAD & 5,000 & $16 \backslash 0.00$ & $16 \backslash 0.00$ & $32 \backslash 0.01$ \\
\hline & 10,000 & $11 \backslash 0.00$ & $14 \backslash 0.00$ & $11 \backslash 0.00$ \\
\hline NONDIA & 5,000 & $15 \backslash 0.00$ & $15 \backslash 0.00$ & $17 \backslash 0.00$ \\
\hline & 10,000 & $15 \backslash 0.02$ & $16 \backslash 0.00$ & $17 \backslash 0.02$ \\
\hline
\end{tabular}


Table 1 (Continued)

\begin{tabular}{|c|c|c|c|c|}
\hline Problems & $\operatorname{Dim}$ & Algorithm 2.1 & PRP+ method & CG-DESCENT method \\
\hline \multirow[t]{2}{*}{$\overline{\text { DQDRTIC }}$} & 5,000 & $17 \backslash 0.00$ & $19 \backslash 0.01$ & $21 \backslash 0.00$ \\
\hline & 10,000 & $26 \backslash 0.01$ & $25 \backslash 0.00$ & $23 \backslash 0.01$ \\
\hline \multirow[t]{2}{*}{ DIXMAANA } & 5,000 & $11 \backslash 0.02$ & $12 \backslash 0.02$ & $16 \backslash 0.00$ \\
\hline & 10,000 & $11 \backslash 0.01$ & $12 \backslash 0.01$ & $14 \backslash 0.02$ \\
\hline \multirow[t]{2}{*}{ DIXMAANB } & 5,000 & $21 \backslash 0.01$ & $20 \backslash 0.02$ & $23 \backslash 0.00$ \\
\hline & 10,000 & $21 \backslash 0.02$ & $20 \backslash 0.02$ & $23 \backslash 0.03$ \\
\hline \multirow[t]{2}{*}{ DIXMAANC } & 5,000 & $22 \backslash 0.01$ & $24 \backslash 0.01$ & $28 \backslash 0.02$ \\
\hline & 10,000 & $22 \backslash 0.01$ & $25 \backslash 0.02$ & $28 \backslash 0.01$ \\
\hline \multirow[t]{2}{*}{ Broyden Tridiagonal } & 5,000 & $77 \backslash 0.02$ & $125 \backslash 0.03$ & $132 \backslash 0.03$ \\
\hline & 10,000 & $76 \backslash 0.04$ & $121 \backslash 0.08$ & $110 \backslash 0.05$ \\
\hline \multirow[t]{2}{*}{ Almost Perturbed Quadratic } & 5,000 & $1,156 \backslash 0.30$ & $1,479 \backslash 0.39$ & $1,448 \backslash 0.31$ \\
\hline & 10,000 & $1,906 \backslash 0.95$ & $2,198 \backslash 1.19$ & $2,149 \backslash 0.92$ \\
\hline \multirow[t]{2}{*}{ Tridiagonal Perturbed Quadratic } & 5,000 & $1,489 \backslash 0.37$ & $1,783 \backslash 0.53$ & $1,562 \backslash 0.41$ \\
\hline & 10,000 & $1,140 \backslash 1.15$ & $1,879 \backslash 1.11$ & $2,477 \backslash 1.25$ \\
\hline \multirow[t]{2}{*}{ EDENSCH } & 5,000 & $325 \backslash 0.04$ & $362 \backslash 0.05$ & $2,157 \backslash 0.33$ \\
\hline & 10,000 & $1,106 \backslash 0.26$ & $1,492 \backslash 0.45$ & $1,594 \backslash 0.47$ \\
\hline \multirow[t]{2}{*}{ VARDIM } & 5,000 & $34 \backslash 0.00$ & $46 \backslash 0.02$ & $46 \backslash 0.00$ \\
\hline & 10,000 & $47 \backslash 0.02$ & $52 \backslash 0.03$ & $52 \backslash 0.03$ \\
\hline \multirow[t]{2}{*}{ LIARWHD } & 5,000 & $24 \backslash 0.01$ & $29 \backslash 0.02$ & $34 \backslash 0.00$ \\
\hline & 10,000 & $28 \backslash 0.01$ & $38 \backslash 0.01$ & $41 \backslash 0.02$ \\
\hline \multirow[t]{2}{*}{ Diagonal 6} & 5,000 & $9 \backslash 0.00$ & $9 \backslash 0.00$ & $9 \backslash 0.00$ \\
\hline & 10,000 & $9 \backslash 0.01$ & $9 \backslash 0.02$ & $9 \backslash 0.01$ \\
\hline \multirow[t]{2}{*}{ DIXMAANG } & 5,000 & $1,186 \backslash 0.42$ & $715 \backslash 0.39$ & $660 \backslash 0.33$ \\
\hline & 10,000 & $2,261 \backslash 1.84$ & $1,275 \backslash 1.42$ & $1,042 \backslash 1.07$ \\
\hline \multirow[t]{2}{*}{ DIXMAANI } & 5,000 & $524 \backslash 0.38$ & $850 \backslash 0.47$ & $702 \backslash 0.36$ \\
\hline & 10,000 & $658 \backslash 1.15$ & $1,266 \backslash 1.37$ & $985 \backslash 1.00$ \\
\hline \multirow[t]{2}{*}{ DIXMAANJ } & 5,000 & $770 \backslash 0.35$ & $829 \backslash 0.44$ & $770 \backslash 0.38$ \\
\hline & 10,000 & $1,823 \backslash 1.19$ & $1,108 \backslash 1.14$ & $1,108 \backslash 1.09$ \\
\hline \multirow[t]{2}{*}{ DIXMAANK } & 5,000 & $1,510 \backslash 0.52$ & $715 \backslash 0.41$ & $812 \backslash 0.41$ \\
\hline & 10,000 & $3,658 \backslash 2.03$ & $963 \backslash 1.09$ & $1,303 \backslash 1.31$ \\
\hline \multirow[t]{2}{*}{ ENGVAL1 } & 5,000 & $5,277 \backslash 0.55$ & $7,474 \backslash 0.86$ & $6,436 \backslash 0.69$ \\
\hline & 10,000 & $7,380 \backslash 1.72$ & $7,196 \backslash 1.62$ & $21,402 \backslash 4.56$ \\
\hline \multirow[t]{2}{*}{ COSINE } & 5,000 & $30 \backslash 0.02$ & $33 \backslash 0.02$ & $31 \backslash 0.03$ \\
\hline & 10,000 & $30 \backslash 0.03$ & $34 \backslash 0.04$ & $31 \backslash 0.03$ \\
\hline \multirow[t]{2}{*}{ DENSCHNB } & 5,000 & $10 \backslash 0.01$ & $14 \backslash 0.02$ & $13 \backslash 0.02$ \\
\hline & 10,000 & $10 \backslash 0.00$ & $15 \backslash 0.00$ & $13 \backslash 0.00$ \\
\hline \multirow[t]{2}{*}{ DENSCHNF } & 5,000 & $19 \backslash 0.01$ & $39 \backslash 0.01$ & $35 \backslash 0.01$ \\
\hline & 10,000 & $19 \backslash 0.02$ & $29 \backslash 0.02$ & $31 \backslash 0.02$ \\
\hline \multirow[t]{2}{*}{ SINQUAD } & 5,000 & $515 \backslash 0.51$ & $976 \backslash 0.83$ & $566 \backslash 0.47$ \\
\hline & 10,000 & $2,011 \backslash 2.61$ & $2,116 \backslash 3.59$ & $5,989 \backslash 10.08$ \\
\hline
\end{tabular}

evaluations, of the M1 method was smaller than the CPU time, or the number of iterations of the M2 method, respectively. In order to estimate the whole effect, we apply the performance profiles of Dolan and Moré [21] in CPU time. From Table 1, some CPU times are zero. In order to have a comprehensive evaluation of the M1 and M2 methods in CPU time, we take the average value of the CPU time for each method, and denote av(M1), av(M2). Then we take the CPU time of each problem plus the average value of av(M1) and av(M2). According to their description, the top curve is the method that solved the most problems in a time that was within a factor $\tau$ of the best time; see Figure 1 and Figure 2. Using the same method, we also test on the number of function evaluations; see Figure 3 and Figure 4.

Obviously, Algorithm 2.1 is competitive to the PRP+ method and the CG-DESCENT method in the number of function evaluations and CPU time. Thus, it is of great importance to study Algorithm 2.1. 


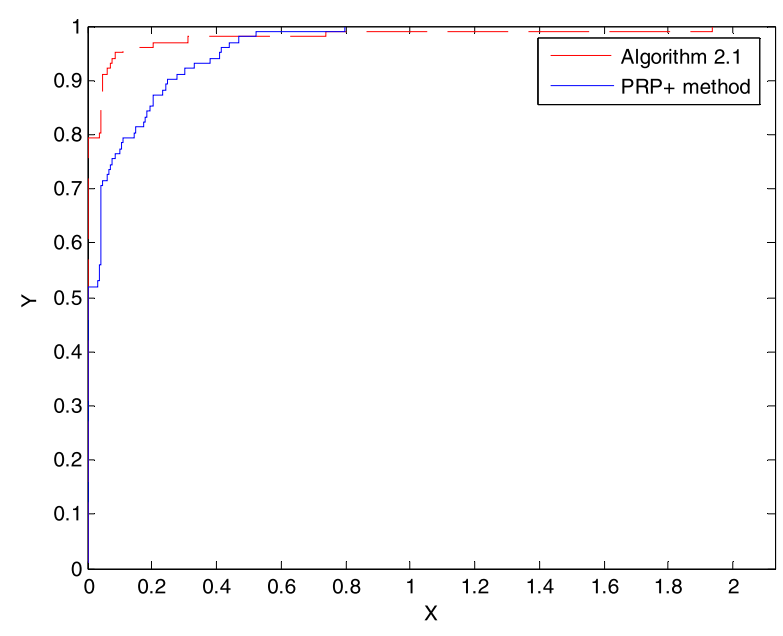

Figure 1 Performance profiles with respect to CPU time in seconds.

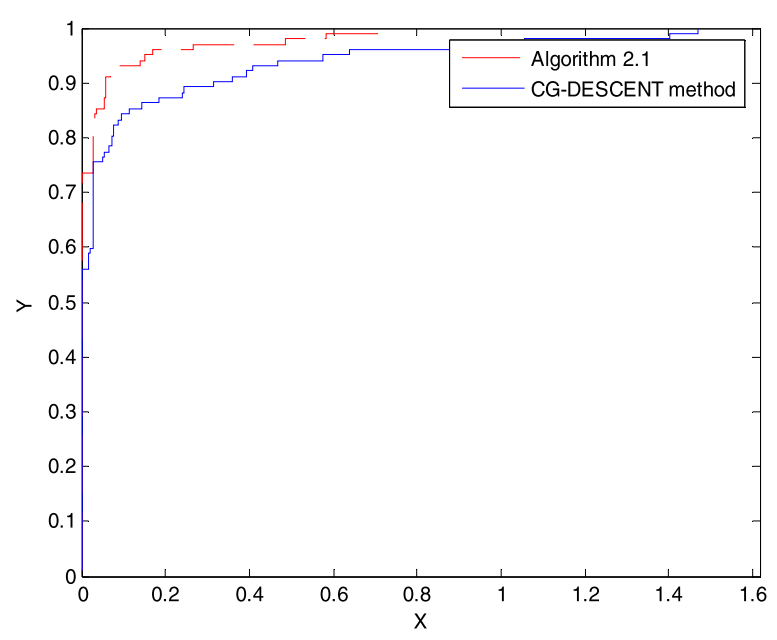

Figure 2 Performance profiles with respect to CPU time in seconds.

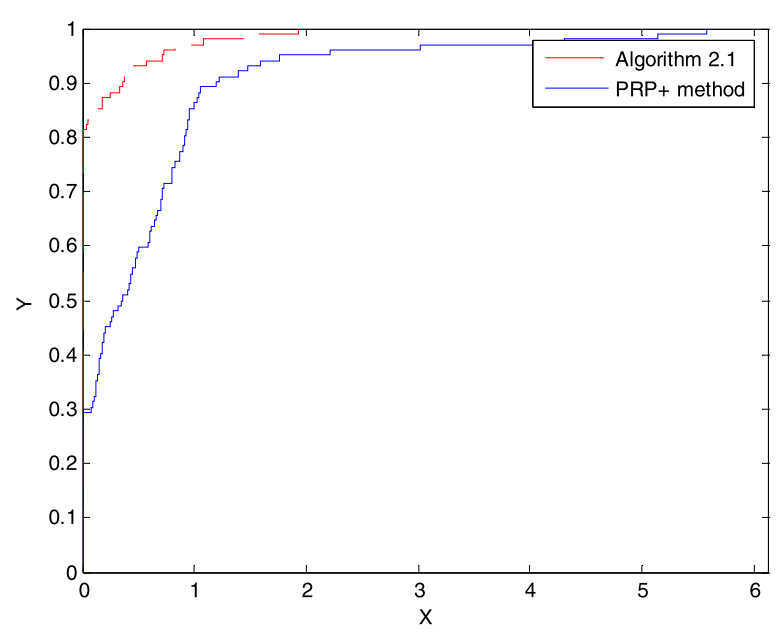

Figure 3 Performance profiles with respect to the numbers of iterations. 


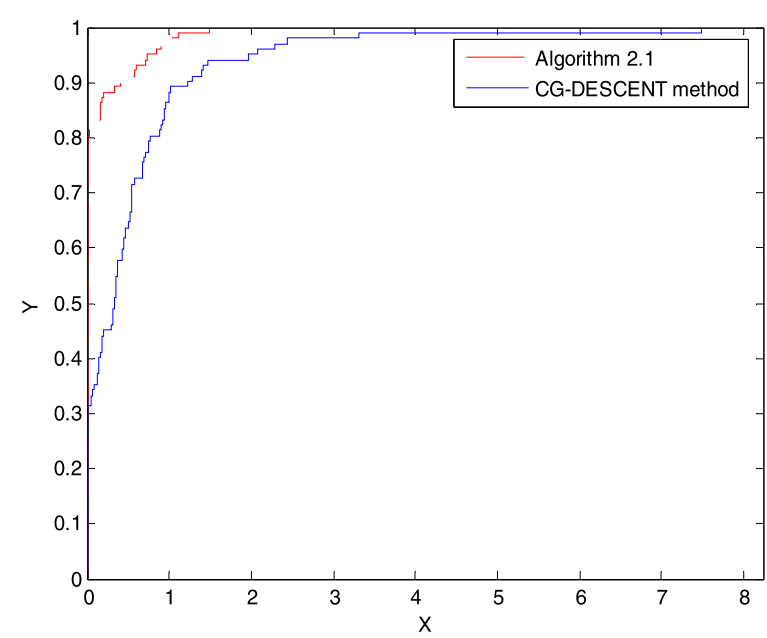

Figure 4 Performance profiles with respect to the numbers of iterations.

\section{Competing interests}

The author declares that she has no competing interests.

\section{Acknowledgements}

The author wishes to express their heartfelt thanks to the anonymous referees and the editor for their detailed and helpful suggestions for revising the manuscript.

\section{Received: 19 March 2014 Accepted: 4 June 2014 Published: 18 Jul 2014}

\section{References}

1. Polak, E, Ribière, G: Note sur la convergence de méthodes de directions conjuguées. Rev. Fr. Inform. Rech. Oper. 3(16), 35-43 (1969)

2. Polak, BT: The conjugate gradient method in extreme problems. USSR Comput. Math. Math. Phys. 9, 94-112 (1969)

3. Liu, Y, Storey, C: Efficient generalized conjugate gradient algorithms. Part 1: theory. J. Optim. Theory Appl. 69, 129-137 (1992)

4. Hager, WW, Zhang, H: A new conjugate gradient method with guaranteed descent and an efficient line search. SIAM J. Optim. 16, 170-192 (2005)

5. Powell, MJD: Nonconvex minimization calculations and the conjugate gradient method. In: Numerical Analysis Lecture Notes in Mathematics, vol. 1066, pp. 122-141. Springer, Berlin (1984)

6. Hager, WW, Zhang, H: A survey of nonlinear conjugate gradient methods. Pac. J. Optim. 2, 35-58 (2006)

7. Zhang, L, Zhou, W, Li, DH: A descent modified Polak-Ribière-Polyak conjugate gradient method and its global convergence. IMA J. Numer. Anal. 26, 629-640 (2006)

8. Li, ZF, Chen, J, Deng, NY: A new conjugate gradient method and its global convergence properties. Math. Program. 78, 375-391 (1997)

9. Liu, J: Convergence properties of a class of nonlinear conjugate gradient methods. Comput. Oper. Res. 40, 2656-2661 (2013)

10. Liu, J, Du, X: Global convergence of a modified LS method. Math. Probl. Eng. 2012, Article ID 910303 (2012)

11. Li, M, Chen, Y, Qu, A-P: Global convergence of a modified Liu-Storey conjugate gradient method. U.P.B. Sci. Bull., Ser. A 74, 11-26 (2012)

12. Tang, C, Wei, Z, Li, G: A new version of the Liu-Storey conjugate gradient method. Appl. Math. Comput. 189, 302-313 (2007)

13. Liu, J, Du, X, Wang, K: Convergence of descent methods with variable parameters. Acta Math. Appl. Sin. 33, 222-230 (2010) (in Chinese)

14. Wei, Z, Yao, S, Liu, L: The convergence properties of some new conjugate gradient methods. Appl. Math. Comput. $183,1341-1350$ (2006)

15. Zoutendijk, G: Nonlinear programming, computational methods. In: Abadie, J (ed.) Integer and Nonlinear Programming, pp. 37-86. North-Holland, Amsterdam (1970)

16. Li, ZF, Chen, J, Deng, NY: Convergence properties of conjugate gradient methods with Goldstein line searches. J. China Agric. Univ. I(4), 15-18 (1996)

17. Dai, YH, Yuan, Y: Nonlinear Conjugate Gradient Method. Shanghai Scientific \& Technical Publishers, Shanghai (2000) (in Chinese)

18. Powell, MJD: Convergence properties of algorithms for nonlinear optimization. SIAM Rev. 28, 487-500 (1986)

19. Bongartz, I, Conn, AR, Gould, NIM, Toint, PL: CUTE: constrained and unconstrained testing environments. ACM Trans. Math. Softw. 21, 123-160 (1995)

20. Andrei, N: An unconstrained optimization test functions collection. Adv. Model. Optim. 10, 147-161 (2008)

21. Dolan, ED, Moré, JJ: Benchmarking optimization software with performance profiles. Math. Program. 91, 201-213 (2002) 
10.1186/1029-242X-2014-248

Cite this article as: Wu: Global convergence of a modified conjugate gradient method. Journal of Inequalities and Applications 2014, 2014:248

Submit your manuscript to a SpringerOpen ${ }^{\circ}$ journal and benefit from:

- Convenient online submission

- Rigorous peer review

- Immediate publication on acceptance

Open access: articles freely available online

- High visibility within the field

- Retaining the copyright to your article

Submit your next manuscript at $\gg$ springeropen.com 